\title{
Nano-pharmaceuticals in Medicine
}

\author{
YOGINDER K GUPTA*,1 and A K DINDA ${ }^{2}$ \\ ${ }^{1}$ Dept. of Pharmacology, All India Institute of Medical Science, New Delhi 110 029, India \\ ${ }^{2}$ Dept. of Pathology, All India Institute of Medical Science, New Delhi 110 029, India
}

(Received on 04 September 2017; Accepted on 05 December 2017)

\begin{abstract}
Nanotechnology is an emerging field with a major goal to target drug delivery to improve the efficacy and safety profile. The application of nanotechnology to medicine, known as nano-medicine, concerns the use of precisely engineered materials to develop novel therapeutic and diagnostic modalities. Nanomaterials possess various unique physiochemical properties, such as small size, large surface area to mass ratio, and high reactivity, which are different from bulk materials. These properties can be used to overcome limitations found associated with traditional therapeutic and diagnostic agents. This review focuses on recent progress and development in the field nano-pharmaceuticals for various biomedical applications.
\end{abstract}

Keywords: Nano-pharmaceuticals; Nano-particles; Nano-medicine; Nanomaterials

\section{Introduction}

Application of nanotechnology in medicine or Nanomedicine is revolutionising the medical practice both in the areas of diagnostics and therapy. One of the major areas of focus in nanomedicine is the area of nanoparticle based drug delivery systems (DDS). These nano DDS can be broadly grouped as nanopharmaceuticals which may have a potential to replace the market of conventional drugs (pharmaceuticals). The drug or other biomolecules can be loaded inside nanoparticles (NPs) which can be targeted to disease site leading to increased bioavailability, therapeutic efficacy simultaneously reducing the off-target effect and toxicity. Over the last several decades, numerous nanocarriers have been developed that include liposomes, polymeric particles, drug conjugates, dendrimers, solid lipid nanoparticle, protein and carbohydrate as well as inorganic system like iron, carbon, silica and so on. The biocompatible and biodegradable carriers hold great potential to be used in drug delivery applications.

In the past few decades, several therapeutics based on nanoparticles, which are particles in the size range of 1-1000 nm, has been successfully introduced for the treatment of various diseases. These therapeutics harness the potential of various opportunities provided by nanomaterials to target the delivery of drugs more precisely, in order to improve their solubility, to extend their half-life, to improve their therapeutic index and to reduce their immunogenicity. The developments made in the field of NPs-based delivery systems have opened up new vistas to engineer non-immunogenic and targeted systems for delivery of biomolecules. Hence, in this report, we focus on the advances made during last five years in India.

\section{Nanoformulations for Vaccines Delivery}

Vaccination has a major impact on the control of infectious diseases. However, there are still many infectious diseases for which the development of an effective vaccine has been elusive. In many cases, the failure to vaccines is a consequence of the inability of vaccine candidates to evoke appropriate immune responses. Over the past decade nanoscale (size $<1000 \mathrm{~nm}$ ) materials, such as virus-like particles, liposomes, polymeric, nanospheres have received attention as potential delivery vehicles for vaccine antigens which can both stabilize vaccine antigens and act as adjuvants. Importantly, some of these NPs are able to enter antigen-presenting cells by different 
pathways, thereby modulating the immune response to the antigen.

The researchers at AIIMS have developed a novel nanoadjuvant or NPs which can deliver different protein antigens as vaccine. The NPs are made of low molecular weight biocompatible, biodegradable polymer Polycaprolactone coated and stabilized with Pluoronics (Prashant et al., 2014). The average size of the NPs is around $60 \mathrm{~nm}$. The slow degradable polymers stay inside the antigen presenting cell invivo for 4 to 6 months and produce sustained high antibody response with single dose. They have demonstrated the antibody response with Tetanus antigen. The major breakthrough is the capability of the NPs for lymphatic targeting following administration through oral route with higher disseminated antigen presentation as well as higher antibody response than conventional injectable vaccine. Recently, a study by Dinda et al. (2016) reported NPs with $14 \mathrm{KDa}$ polycaprolactone entrapping hepatitis B surface antigen (HBsAg) stabilized with Pluronics ${ }^{\circledR}$ F127 and used it as oral delivery vehicle (Dinda et al., 2016). The efficacy for specific antibody production was evaluated and compared with parenteral routes of immunization in mice. A superior antibody response with a higher titer of anti-HBsAg antibody till 2 months following single oral administration compared to other routes of immunization and conventional alum-based $\mathrm{HBsAg}$ vaccine was found. The NPs with antigen were found in the macrophages residing in small intestinal villi, peripheral lymph nodes and other reticulo-endothelial organs 2 months after oral administration. This study suggests the efficacy of current nanocarrier system for efficient antigen presentation disseminated in peripheral lymphoid tissues, following oral administration with a prolonged antibody response, which can minimize the requirement of booster dose.

In a study by Harde et al. development of a novel NPs, comprising of stable glucomannosylated chitosan, for oral immunization was demonstrated (Harshad et al., 2014). Chitosan nanoparticles were stabilized by tandem cross-linking using tripolyphosphate followed by glutaraldehyde. The in vitro and in vivo performances were established in RAW 264.7 and BALB/c mice, respectively. Results illustrated that the lyophilized formulation was exceptionally stable in simulated biological media and the enclosed antigen was conformationally stable. The mechanistic understanding of glucomannosylated chitosan NPs in RAW 264.7 revealed trans-cellular uptake via both mannose and glucose transportermediated endocytosis. Glucomannan modification resulted in significantly higher systemic (serum IgG titer), mucosal (secretory IgA) and cell-mediated (IL2 and IFN- $\gamma$ ) immune responses in comparison with non-modified chitosan NPs. As a result, the present strategy is expected to contribute some novel tools for the oral delivery of numerous bio-macromolecules.

In another study by Smitha et al. (2014), the development of amidase, an effective vaccine to prevent infections caused by $S$. aureus, encapsulated O-carboxymethyl chitosan NPs (Ami-O-CMC NPs) of $300 \pm 50 \mathrm{~nm}$ was reported. The particles were prepared by ionic cross-linking method. The prepared Ami-O-CMC NPs had an encapsulation efficiency of $55.39 \%$. Haemolysis assay and cytotoxicity studies proved the hemo-compatibility and cyto-compatibility of the prepared NPs. The sustained release of Ami from the NPs is expected to prolong its immunogenicity and in turn leads to development of better protective immunity against Staphylococcus aureus infections.

\section{Nanoformulations for Anti-Tuberculosis Drugs}

Tuberculosis, a highly contagious persistent infection caused by Mycobacterium tuberculosis and Mycobacterium bovis. Tuberculosis is the world's second most common cause of death after HIV/ AIDS. In 1993, World Health Organization declared as a global emergency. The disease typically attacks lungs but can also develop as extra pulmonary TB in the central nervous or circulatory systems or elsewhere in the body. Despite potential curative pharmacotherapies being available for over 50 years, the length of treatment and pill burden can hamper patient lifestyle. Thus, low compliance and adherence to administration schedules remain the major reasons for therapeutic failure and contribute to the development of multi-drug-resistant strains. NPs based delivery systems are suitable for targeting chronic diseases such as tuberculosis as these cross the biological barriers and target cellular reservoirs of Mycobacterium tuberculosis. Experimental data supports the possibility of intermittent chemotherapy with key first-line as well as second-line anti- 
tubercular drugs by employing synthetic or natural carriers, chiefly polymers.

AIIMS group has developed a novel nanocomposite with low molecular weight Polycaprolactone and gelatin using a layer by layer technology, wherein four anti tubercular drugs (INH, Rifampicin, Ethambutol and Pyrizinamide) can be loaded in different compartments in one NP. This system is being optimised for respiratory and oral delivery systems (Indian Patent Application \#1141/ DEL/2014).

Varma et al. (2015) used pyrazinamide, which is a highly specific agent against Mycobacterium tuberculosis, to formulate polymeric NPs to sustain the release and reduction of the dosing frequency of pyrazinamide (Ravi et al., 2015). The formulated polymeric NPs were in the particle size range of 45$300 \mathrm{~nm}$ with a maximum drug entrapment efficiency of $80.9 \%$. The stability study of optimized batch conducted at $40 \pm 2^{\circ} \mathrm{C} / 75 \pm 5 \%$ relative humidity showed no significant changes up to 90 days. X-Ray Diffraction spectrum exhibits the transformation of crystalline form of drug to amorphous in the formulation. Scanning Electron Microscope image showed that NPs were spherical in shape with smooth surface. In vitro release profiles were biphasic in nature with burst release followed by controlled release over a period of $24 \mathrm{hr}$, obeying the diffusion mechanism. In vivo and ex vivo studies results of this study showed significant uptake of the NPs by alveolar macrophages through fluorescent micrograph. Polymeric NPs formulation of pyrazinamide could encompass significant uptake by alveolar macrophages, the high first-pass metabolism, sustain the release of drug leading to reduction in dose, toxicity and improvement of patient compliance.

Though there are promising results seen in several preclinical animal model of tuberculosis, the greatest challenge is to deliver 4 anti-tubercular drugs in correct therapeutic ratio (Bacteriostatic: Bactericidal) at the disease site. In this regard Prof. Dinda's group in collaboration with Prof. Y.K. Gupta has fabricated a novel nanocarrier with polycaprolactone and gelatin layer by layer loading 4 drugs in one NP with each drug in separate compartment. It showed a high bactericidal activity (Indian Patent Application \#1141/DEL/2014).

\section{Nano-formulations for Cancer Therapy}

Cancer is a rapidly emerging disease nowadays. The use of chemotherapeutic drugs is, however, limited due to the random and non-targeted distribution of these drugs in the body leading to undesirable side effects with subsequent decrease in their therapeutic efficacy. Consequently, site specific delivery of anticancer drugs is the most important requirement for effective chemotherapy. In this regard, scientists have generated great interest in the NPs based delivery systems, owing to their ability to have enhanced penetration and retention effect at the tumor site. Also, targeting the specific cells of the body can be achieved by surface modifications. Several reports have demonstrated the enhanced efficacy of drug loaded NPs in cancer therapeutics.

A study by Kumar et al. (2014), used iron oxide nanoparticles to deliver an oncopeptide, NuBCP-9, targeting the BCL-2 BH3 domain (Kumar et al., 2014). Citric acid/2-bromo 2-methylpropanoic acid (CA/BMPA)-capped super-paramagnetic iron oxide NPs were used to immobilize and deliver the NuBCP9 peptide to the cancer cells without any noticeable off-target effects. Results demonstrated that NuBCP9- superparamagnetic iron oxide NPs efficiently penetrate into cancer cells and bind to its intracellular target protein BCL-2. Moreover, significant inhibition of proliferation and substantial induction of cell death were observed when cancer cells were treated with NuBCP-9- superparamagnetic iron oxide NPs at different time intervals. Importantly, the $\mathrm{IC}_{50}$ values for killing of breast cancer cells with NuBCP-9superparamagnetic iron oxide NPs were much lower compared to cells treated with the NuBCP-9 peptide linked with a CPP (Arg-8; NuBCP-9-R8). Molecular and biochemical analyses further supported that NuBCP-9-SPIONs killed breast cancer cells by apoptosis-mediated mechanisms. Furthermore, the data demonstrated that administration of NuBCP-9SPIONs to mice bearing Ehrlich ascites tumors was associated with loss of tumorigenicity and extensive apoptosis in tumor tissues. Taken together, these findings showed that a non-CPP-tagged peptide can be successfully delivered to undruggable intracellular onco-targets using superparamagnetic iron oxide NPs.

Researchers at Amrita University, Kerala demonstrated unique of nanofiber for the controlled 
delivery of anti-cancer drug, Temozolomide, in orthotopic brain-tumor for one month using flexible polymeric nano-implant (Ramachandran et al., 2017). A library of drug loaded (20 wt \%) electrospun nanofiber of PLGA-PLA-PCL blends with distinct in vivo brain-release kinetics (hours to months) were numerically selected. Orthotopic rat glioma implanted wafers showed constant drug release with negligible leakage into the peripheral blood rendering 1000 fold differential drug dosage in tumor versus peripheral blood. Most importantly, implant with one month release profile resulted in long-term ( $>4$ month) survival of $85.7 \%$ animals, whereas 07 day releasing implant showed tumor recurrence in $54.6 \%$ animals, rendering a median survival of only 74 days. In conclusion, highly controlled drug delivery is possible for prolonged periods in orthotopic brain-tumor using combinatorial nanofibre libraries of bulk-eroding polymers, thereby controlling glioma recurrence.

Transferrin-targeted core-shell Nanomedicine encapsulating two clinically used drugs, doxorubicin and sorafenib were formulated by researchers at Amrita University against liver cancer (Malarvizhi et al., 2014). Doxorubicin loaded Poly(vinyl alcohol) nano-core and sorafenib loaded albumin nano-shells were prepared. Doxorubicin loaded in nano-shell intercalates in DNA killing cancer cells. Whereas, nano-shells release sorafenib inhibited aberrant oncogenic signaling involved in cell proliferation. They showed enhanced cellular uptake synergistic cytotoxicity in $\sim 92 \%$ of cells when iron-deficient microenvironment was present via transferrin ligands targeting. Efficient penetration of targeted core-shell NPs in 3D spheroids of liver tumor causes uniform cell killing. Hence, designed core-shell NPs can be effectively combined with clinically relevant singleagent drugs for exerting synergistic activity against liver cancer.

Bhatnagar et al. (2015) used bromelain (BL), a proteolytic enzyme, an anti-tumor agent which however has been limited due to the requirement of high doses at the tumor site (Bhatnagar et al., 2015). They developed the BL loaded poly (lactic-co-glycolic acid) NPs that show enhanced anti-tumor effects compared to free BL. The formulated NPs with a mean particle size of $130.4 \pm 8.81 \mathrm{~nm}$ exhibited sustained release of BL. Subsequent investigation revealed enhanced anti-tumor ability of NPs in 2-stage skin tumorigenesis mice model. Reduction in average number of tumors ( $\sim 2.3$ folds), delay in tumorigenesis ( $\sim 2$ weeks), percent tumorigenesis ( $\sim 4$ folds), and percent mortality rate, as well as a reduction in the average tumor volume ( 2.5 folds $)$ in mice as compared to free BL were observed. NPs were also capable of modulating the expression of pro-apoptotic $(\mathrm{P} 53, \mathrm{Bax})$ and anti-apoptotic $(\mathrm{Bcl} 2)$ proteins. Therefore, our findings demonstrate that developed NPs formulation could be used to improve the efficacy of chemotherapy by exerting chemo-preventive effects against induced carcinogenesis at lower doses.

Thimmaraju et al. (2017) demonstrated poloxamer based formulations of meloxicam. They evaluated various parameters like $\mathrm{pH}$ stability, drug release and in vitro anti-cancer activities in cell lines with an intention to formulate injectable sustained biodegradable drug delivery system. Various strengths of meloxicam formulations were prepared by using poloxamer 407 and were analyzed for drug content and $\mathrm{pH}$ stability by using HPLC. Drug release studies were tested by using USP dissolution testing apparatus. Further, they evaluated in vitro anti-cancer activity among these formulations by using sulphorhodamine-B assay in two leukemia cell lines such as HL-60 and K-562 cell lines. It also showed $60 \%$ drug release and exhibited good anti-cancer activity in HL-60 cell line with GI50 $\leq 10 \mu \mathrm{g} / \mathrm{ml}$, similar to adriamycin. Thus, the formulation has a potential to be a sustained release, passive targeted delivery system for meloxicam, with reduced side effects associated with the drug.

Researchers at IIT Delhi demonstrated poly (styrene-co-maleic anhydride), a biocompatible copolymer that was further conjugated with paclitaxel (PTX) via ester linkage and self-assembled to form poly (styrene-co-maleic acid)-paclitaxel (PSMACPTX) NPs (Dalela et al., 2015). The in vitro release of PTX from PSMAC-PTX NPs showed a higher release at lower $\mathrm{pH}$ than at the physiological $\mathrm{pH}$ of 7.4 , confirming its $\mathrm{pH}$-dependent release. $\mathrm{IC}_{50}$ values of $9.05-18.43 \mathrm{ng} / \mathrm{mL}$ of PTX equivalent were observed in various cancer cell lines after $72 \mathrm{hr}$ of incubation. Pharmacokinetic studies revealed that the conjugation of PTX to the PSMAC co-polymer not only increased the plasma and tumor $\mathrm{C}(\max )$ of PTX but also prolonged its plasma half-life and retention in tumor via enhanced permeability and retention effect. 
Administration of PSMAC-PTX NPs showed significant tumor growth inhibition with improved apoptosis effects in vivo on Ehrlich Ascites Tumor (EAT)-bearing BALB/c syngeneic mice in comparison with Taxol, without showing any cytotoxicity. Therefore, PSMAC-PTX NPs may be considered as an alternative nanodrug delivery system for the delivery of PTX in solid tumors.

PLGA nanoparticles loaded with 2Methoxyestradiol were prepared and stabilized with casein or poly(ethylene glycol) by Gopikrishna et al., 2017 et al. These particles were evaluated fo cellular interactions, pharmacokinetics and tumour accumulation. It was found that particle-cell interactions and the anti-angiogenesis activity were similar for both nanoformulations in vitro. But in case of PEGylated nanoformulation, the in vivo pharmacokinetics and tumour accumulation of the drug were substantially improved. Reduced protein binding was observed for PEG stabilized PLGA NPs. Hence, 2-Methoxyestradiol nanoencapsuled with PEGylated PLGA nanocarrier could improve its halflife and plasma concentration and thereby increase the tumour accumulation.

\section{Nanoformulations for Ocular Delivery}

The unique anatomy and physiology of eye poses a huge challenge to the delivery of drugs in the eye. The blinking reflex and tear drainage prevents drug retention on ocular surface which necessitates repeated drug dosing; this affects patient compliance, causes blurring of vision etc. Moreover, the different anatomical layers of the eye prevent topical drugs from reaching the posterior segment of the eye. Over the last decade, the benefits of nanotechnology in encapsulating drugs in NPs for sustained release and improved availability has made remarkable contribution towards ocular drug delivery.

Posterior capsular opacification (PCO) is a major post-surgical complication following cataract surgery. Different approaches to prevent PCO have been attempted by targeting the cells involved in the pathology or by targeting molecular pathways involved in this fibrotic change. Major cause of failure with intraocular drugs was either toxicity or inadequate drug availability. Sustained delivery of Doxorubicin through Methyl polyethylene glycol-polycaprolactone NPs was designed to selectively destroy lens epithelial cells after cataract surgery to avoid toxicity induced by Doxorubicin on the healthy cells of the eye (Guha et al., 2013). In vitro evaluation of cellular entry of Doxorubicin NPs and free drug and arrest of cellular proliferation was evaluated in lens epithelial cells. In vivo evaluation of efficacy of Doxorubicin NPs in prevention of PCO and its safety for ocular use was reported in rabbit model.

Curcumin has been established for its antiinflammatory, anti-angiogenic and anti-fibrotic property, but unfortunately major hindrance in exploiting the properties of curcumin for clinical use is its insolubility and poor bioavailability. To improve the retention time and bioavailability of Curcumin, curcumin loaded NPs were designed and evaluated in animal model of corneal neovascularisation (CNV) (Nirparaj et al., 2015). CNV is a leading cause for compromised vision. Therapeutic prevention of $\mathrm{CNV}$ is a major clinical challenge and there is a compelling need to seek effective and safe therapy for this pathology. MePEG-PCL NPs of curcumin has shown increased efficiency in preventing angiogenic sprouting in vitro. Topical delivery of curcumin NPs in the eye showed enhanced retention of curcumin in the cornea, significant improvement in prevention of $\mathrm{CNV}$ over free curcumin as graded clinically and was supported by data showing suppression of VEGF, inflammatory cytokines and MMP expression. Anti-inflammatory activity of curcumin was demonstrated by suppression of NFKB. Histopathology and scanning electron microscopy shows absence of any adverse change in the corneal structure following application of curcumin NPs.

\section{Nanoformulations for Targeting Central Nervous System}

\section{Alzeimer's Disease}

Neurogenesis is a process of generation of new neurons which is negatively regulated by age, stress, sleep deprivation and increased by caloric restriction, exercise, and physiological activation. Several animal and clinical studies suggest reduced neurogenesis in neurodegenerative disorders such as Parkinson's disease, Alzheimer's disease, and Huntington's disease. Hence, nanoparticles mediated induction of neurogenesis via targeting endogenous neural stem could be a promising therapeutic approach to such diseases. 
Tiwari et al. (2014), working at done at IITR, Lucknow, loaded curcumin, a neuroprotective agent with limited brain bio-avlailability, in PLGA formulating NPs. Better induction of neural stem cell proliferation and neuronal differentiation in vitro and in the hippocampus and subventricular zone of adult rats was observed as compared to bulk curcumin. The NPs were also found to significantly increase the expression of genes involved in cell proliferation (reelin, nestin, and Pax6) and neuronal differentiation (neurogenin, neuroD1, neuregulin, neuroligin, and Stat3). Reverse learning and memory impairments in an amyloid beta induced rat model of AD-like phenotypes, by inducing neurogenesis by these NPs was also observed. Molecular docking studies suggest curcumin interaction with Wif-1, Dkk, and GSK-33. Hence, curcumin NPs could provide an interesting therapeutic approach to neurodegenerative diseases such as Alzeimer's disease by inducing brain selfrepair mechanism.

\section{Parkinson's Disease}

Pahuja et al. (2015) demonstrated sustained and safe delivery of dopamine across the blood brain barrier for successful therapy in Parkinson's disease was. Dopamine-loaded PLGA NPs (DA NPs) were synthesized to deliver dopamine to the brain. These NPs slowly and constantly released dopamine, showed reduced clearance of dopamine in plasma, reduced quinone adduct formation, and decreased dopamine autoxidation. DA NPs were internalized in dopaminergic SH-SY5Y cells and dopaminergic neurons in the substantia nigra and striatum, regions affected in Parkinson's disease. NPs were able to cross the BBB and capillary endothelium in the striatum and substantia nigra in a 6-hydroxydopamine induced rat model of Parkinson's disease. Systemic intravenous administration of DA NPs caused significantly increased levels of dopamine and its metabolites and reduced dopamine-D2 receptor supersensitivity in the striatum of parkinsonian rats. These results suggest that NPs delivered dopamine into the brain, reduced dopamine autoxidationmediated toxicity, and ultimately reversed neurochemical and neurobehavioral deficits in parkinsonian rats.

\section{Epilepsy}

Benzodiazepine class of drugs are widely used as antiepileptic drugs. Diazepam, lorazepam and midazolam are given via intravenous route as first line of treatment in the emergency management of Status Epilepticus. In a study by Sharma et al. a prehospital treatment in the form of a delivery system which could be given via non-invasive route (nasal route) to the patients suffering from SE in emergency conditions was demonstrated. PLGA NPs of Diazepam, lorazepam and midazolam were prepared via nano-precipitation method for nose to brain delivery (Sharma et al., 2016, Sharma et al., 2015). The developed NPs were found to be in nanometric range with high percentage drug entrapment, which was further confirmed by TEM imaging. In vitro and $e x$ vivo drug release supported the hypothesis of fast onset of action from PLGA NPs by showing initial burst release and further controlled drug release. Cell viability assay on Vero cell line showed that developed drug loaded NPs were not cytotoxic. The biodistribution studies on Sprague-Dawley rats with technetium-99m radiolabelled drugs supported the hypothesis of nose to brain pathway for drug delivery of developed polymeric NPs. The results showed that drug loaded polymeric NPs showed higher brain uptake of drug when administrated intra-nasally compared to intranasal drug solution and intravenous drug solution. Pharmacokinetic studies suggested prolonged action of diazepam/midazolam/lorazepam concentrations in brain (higher $\mathrm{AUC}_{\text {Brain }}$ ) due to slow erosion of PLGA from NPs. Brain/blood distribution studies showed that drug loaded polymeric NPs provided higher brain/blood concentration of drug after NPs intranasal administration, compared with drug solution intranasal and intravenous administration. Moreover, Gamma Scintigraphic images were in agreement with the bio-distribution results in SpragueDawley rats. The investigation demonstrated that intranasal drug loaded NPs can potentially transport diazepam, midazolam and lorazepam via nose-to-brain and can serve as a non-invasive alternative for prehospital treatment of SE.

\section{Nanoformulations for Wound Healing}

Wound healing is an innate physiological response that helps restore cellular and anatomic continuity of a tissue. Selective biodegradable and biocompatible polymer materials have provided useful scaffolds for wound healing and assisted cellular messaging. Nanotechnology is on the threshold of providing a host 
of new materials and approaches, revolutionizing the medical and pharmaceutical fields. Recently, silver NPs have attracted interest for a clinical application because of its potential biological properties such as anti-bacterial, anti-inflammatory, and also wound healing efficacy, which could be exploited in developing better dressings for wounds and ulcers.

Researchers at AIIMS developed Silver nanoparticles $(20-30 \mathrm{~nm}$ in size) loaded inside Glutathione Collagen NPs which showed a higher antiinfective and wound healing property in comparison to silver sulpha diazene (Indian Patent Application \#201711007245). The silver NPs embedded inside the collagen NPs are less toxic and the anti-microbiocidal activity is due to silver ions released at the wound area.

Chitosan alginate mucoadhesive NPs were fabricated for treatment of pemphigous ulcers and other ulcers in mouth. These particles were loaded with different immunosuppressive and antiinflammatory agents for local sustained release at the mucosal ulcer bed. Both the systems can be modified for application to periodontal infection and other inflammatory diseases.

Dhapte et al. (2014) developed biogenic silver NPs, using a single-step green synthesis of silver NPs (AgNPs) to investigate in vitro and in vivo behaviour as wound-healing agents. Stable AgNPs with characteristic absorption at $408 \mathrm{~nm}$ and $15 \pm 3 \mathrm{~nm}$ particle size were generated via the active involvement of Bryonia laciniosa. No loss of stability was detected after 6 months at room temperature. Anti-bacterial activity was observed against both Gram-negative and Gram-positive bacteria with no cytotoxicity observed in vitro at a concentration of $200 \mu \mathrm{g} / \mathrm{mL}$ and effective cytokine modulation. In vivo wound healing experiments showed improved wound contracting ability in rats where, after 14 days, wound alleviation was $47.1 \pm 2.2 \%$ in the control groups, compared with $78.1 \pm 1.4 \%$ and $92.6 \pm 6.7 \%$ for a silver-based marketed cream and the AgNPs, respectively. As a result, the developed AgNPs proved to be superior wound healing agents owing to scarless healing with insignificant inflammation and toxicity.

Tiwari et al. (2014) illustrated the synthesis of copper NPs by Pseudomonas aeruginosa and testing their efficacy in enhancing the pace of wound healing.
Culture supernatant was used to synthesise copper NPs. Optimum conditions were selected to maximise the biosynthesis of NPs. Biosynthesised copper NPs (BNCPs) were characterised by Malvern zeta sizer and scanning electron microscopy. Average particle size, polydispersity index and zeta potential of BNCPs were found to be $110.9 \mathrm{~nm}, 0.312$ and (-) $18.3 \mathrm{mV}$, respectively. BNCPs were evaluated for its wound healing activity by excision wound model in rat. The pace of wound healing was enhanced by BNCPs compared with copper in native form.

\section{Nanoformulations for Anti-leishmaniasis Drugs}

Visceral leishmaniasis is a fatal vector-borne parasitic syndrome attributable to the protozoa of the Leishmania donovani complex. The available chemotherapeutic options are not ideal due to their potential toxicity, high cost and prolonged treatment schedule.

Want et al. (2015) used nano drug delivery systems for plant-derived secondary metabolite, artemisinin, as an alternative strategy for the treatment of experimental visceral leishmaniasis (Want et al., 2015). Artemisinin-loaded poly (lactic-co-glycolic acid) (ALPLGA) NPs prepared were spherical in shape with a particle size of $220.0 \pm 15.0 \mathrm{~nm}, 29.2 \pm 2.0 \%$ drug loading and $69.0 \pm 3.3 \%$ encapsulation efficiency. ALPLGA NPs administered showed superior antileishmanial efficacy compared with free artemisinin in BALB/c model of visceral leishmaniasis. There was a significant reduction in hepatosplenomegaly as well as in parasite load in the liver $(85.0 \pm 5.4 \%)$ and spleen $(82.0 \pm 2.4 \%)$ with ALPLGA nanoparticles treatment at $20 \mathrm{mg} / \mathrm{kg}$ body weight compared to free artemisinin $(70.3 \pm 0.6 \%$ in liver and $62.7 \pm 3.7 \%$ in spleen). In addition, ALPLGA NPs treatment restored the defective host immune response in mice with established visceral leishmaniasis infection. In conclusion, results advocate superior efficacy of ALPLGA NPs over free artemisinin, which was coupled with restoration of suppressed cell-mediated immunity in animal models of visceral leishmaniasis.

Dr. Dinda's group in collaboration with Prof. Y.K.Gupta, at AIIMS, New Delhi, fabricated a low cost cholesterol grafted chitosan NPs stabilised by Pluronics with a high (>65\%) Amphotericin B entrapment efficiency. This system showed 10 fold higher efficacy than Ambisome and also effective 
through oral administration in rodent model (Dinda $e t$ al., 2016).

\section{Nanoformulations for Anti-fungal Drugs}

AIIMS group has fabricated Chitosan grafted cholesterol NPs with average size of $220 \mathrm{~nm}$ with a high (63\%) loading efficiency of Amphotericin B. It has an unique property of endosomal escape in parasite containing cells leading to sustained cytosolic release of drug leading to higher parasitocidal activity in 1/10 dose of Amphotericin B when compared with the liposomal preparation Ambisome. This nanocarrier can deliver Amphotericin B orally as documented in rodent model. This novel low cost nanocarrier for Amphotericin B with higher efficacy and low toxicity has a high translational potential (Indian Patent Application \#201611009768).

\section{Nanoformulation for Cosmeceuticals}

Researchers at AIIMS have fabricated a novel Glutathione and goat Collagen NPs which has high antioxidant and pro-healing property (Indian Patent Application \#201711007245). It can reduce melanin synthesis following sun exposure (UV irradiation). The average size of NPs was found out to be $30 \mathrm{~nm}$. It can be loaded with curcumin and other agents with a high potential use for nano-cosmeceuticals.

\section{Nanocarriers for Oral Gene Delivery}

Gene or any other nucleic acid (Micro RNA/ SiRNA) cannot be delivered orally due to acid peptic digestion. A group at AIIMS, New Delhi have loaded the DNA (gene) in calcium phosphate NPs and sequentially coated it with Poly acrylic acid (PAA), Chitosan and PAA. This novel layer by layer technology makes particle acid resistant resulting in successful oral gene delivery system as demonstrated in murine model (Indian Patent application \# 3256/DEL/2012).

\section{Nanoformulations for Anti-Bacterial Drugs}

Blood borne bacterial infections continue to be a major cause of mortality despite significant advances in modern medicine. Most bacterial infection causes fever of unknown origin without any unique clinical symptoms; these cases require diagnostic confirmation and appropriate antibiotic therapy should be administered. Culture diagnosis has been the gold standard in bacterial infection diagnosis for last 75 years. In many of the infection episodes, the time lost in diagnosis can be a major cause of mortality. Requirement for a rapid diagnosis has been stressed by many health/funding agencies across the world. Hence with nanotechnology components/devices, many interesting technical advances in molecular diagnostics, nucleic acid amplification assays have been developed.

Researchers at IIT have developed a novel typhoid diagnostic system called iMC2 (Singh et al., 2016) with a reduced turnaround time of $7 \mathrm{hr}$. Antibody coated NPs to specifically isolate target cells from a large sample volume by using a unique combination of magnetic field was used. Blood sample was collected in a capture bottle with prefilled reagents and incubated for $6 \mathrm{hr}$. After the incubation period, target cells were isolated from bulk sample and enriched by 200 folds. This enrichment allows detection of Salmonella typhi cells using lateral flow assay strip with reduced time.

Further, reduced the turnaround time for bacterial detection to 60 minutes using SiMED (Spot immunomagnetic enrichment device) (Singh et al., 2016). Magnetic NPs were used to tag the target pathogens and captured into a $100 \mathrm{ml}$ volume chamber using moving magnetic field. By combing immuno-magnetic enrichment with fluorescent detection, they have been able to identify ultralow concentrations of typhi cells with a turnaround time of an hr. These novel applications of nanomaterial will continue to improve the performance of diagnostics, healthcare applications.

\section{Nanoformulations as Biomarker}

One of the most important factors in electrochemical immunosensing is the quality of sensing electrode. During the development of highly sensitive and stable immunosensors, it is a crucial requisite to create new types of electrodes that allow fast and simple measurements of specific biological interactions. Organic self-assembled monolayers are of great significance in surface technology since the presence of chemically bound molecules render the properties of an interface entirely different compared to those that are unmodified. The most commonly used biomarker for Prostate cancer detection is Prostate specific antigen. In a study done at MGPG College, Gorakhpur, gold screen printed electrode were 
modified and functionalized by Self Assembled Monolayer of cysteamine and Single Walled Carbon nanotubes (SWNTs) for detection of Prostate specific antigen (Shukla et al., 2017, Guiseppi et al., 2010). The Prostate specific antigen immobilized electrode was placed in a three electrode cell as working electrode. DPV measurements were used to determine the linear range and to estimate the detection limit of Prostate specific antigen as the routine biosensor. The voltammograms clearly showed the plot of the peak current versus Prostate specific antigen concentration of cysteamine, cysteamine/ SWNTs, cysteamine/ SWNTs/Anti- Prostate specific antigen modified SPE. By modification of cysteamine/ SWNTs, a well-defined response was observed as compared to cysteamine modified SPE. The cysteamine and SWNTs modified gold screen printed electrode will propound new area in the field of Biosensors used for Prostate cancer detection.

\section{Nanoformulations for Multiple Release}

To achieve multiple release profiles, NPs with complex architectures, such as those with multiple shells responding to the trigger to different extents or those which respond to different triggers are engineered. Though promising, the complex chemistries involved in their synthesis is challenging. Towards this, group of researchers at IIT, Delhi developed a simple formulation using a mixture of NPs, responding to different extents for a single trigger, temperature (Deshpande et al., 2017). The formulation consists of Gold NPs based core with a thermoresponsive polymeric shell composed of pNIPAm or pNIPMAm, able to deliver the drugs using capacitive radiofrequency generated hyperthermia. Exposing the system to radiofrequency for shorter time resulted into increase of the temperature, consequently resulting into release of drug primarily from pNIPAm shells, while the second exposure, for longer time, resulted into release from pNIPMAm shells. Thus, it provides a simple alternative for developing a sustained-triggered drug delivery system.

\section{Nanoformulations from Herbal extracts}

Researchers at Delhi University have developed silver NPs (AgNPs) using plant extract of various medicinal plants and conducted bioassay against bacteria, cancer and various mosquito vectors. The NPs were synthesized using tannic acid and determined their anti-bacterial activity against both gram positive (Staphylococcus aureus) and gram negative (Escherichia coli and Pseudomonas aeruginosa) bacteria. The mean size of AgNPs was $12 \mathrm{~nm}$ and stable up to seven months. It was reported that AgNPs at low concentration exhibited strong anti-bacterial activity (Gupta et al., 2013).

Also, AgNPs were synthesized using bark extract of Holarrhena antidysenterica and evaluated larvicidal activity against third instar larvae of $A$. stephens. It has been reported that green synthesized AgNPs exhibited strong larvicidal activity against A. stephens having $\mathrm{LC}_{50}$ and $\mathrm{LC}_{90}$ value of $2.672 \mathrm{ppm}$ and 4.482 ppm only, respectively (Kumar et al., 2017).

\section{Nanoformulations for Peptide Delivery}

Consistent efforts in developing effective nanopharmaceuticals in the Srivastava lab at IISER Bhopal, leads to development of biocompatible and biodegradable polyelectrolytes derived from polyaspartic acid framework (Sharma et al., 2013). The muco-adhesive nature of the polyamide backbone of these polyelectrolytes is noteworthy. Electrostatic interactions between oppositely-charged polyelectrolytes yielded nano sized complexes that were stabilised through in-situ disulphide crosslinking. The disulphide cross linking stabilised these nanocomplexes under physiological conditions but made them susceptible to reducing conditions such as those found intra-cellularly. These redox-responsive nano complexes were utilised for facile encapsulation of the peptide therapeutics such as Insulin and its delivery to lung cancer cells. Once internalised, these nano complexes unravelled to release the entrapped peptide cargo intra-cellularly.

This achievement was further elaborated by developing amphiphilic polymers towards delivering hydrophobic drugs such as Curcumin (Fatima et al., 2016). Facile solubilisation of commercial Curcumin powder in aqueous medium was achieved by utilising these polymers. Very high drug loading (upto $40 \mathrm{wt} . \%$ ) was also possible in these nano-formulations. The inherent hemotoxicity of the polymers was significantly reduced by polyelectrolyte complexation with sodium polyaspartate. The resulting nano-formulations had a favourable size and could be dehydrated to yield reconstitutable powders. 


\section{Nanoformulations for Gene Delivery}

Researchers at IIT Guwahati demonstrated cationic BSA nanoparticle system incorporating bimetallic $\mathrm{Au}-$ $\mathrm{Ag}$ luminescent nanoclusters (NCs) for the delivery of therapeutic suicide gene in HeLa cancer cells (Deepanjalee et al., 2016). Combinatorial therapy is achieved with cationic BSA Au-Ag NCs composite NPs loaded with pDNA, which binds efficiently with pDNA due to its positive nature. While successful delivery of a suicide gene (CD-UPRT) into cells by pDNA loaded composite NPs initiates a therapeutic response cascade by converting prodrug 5-FC to 5$\mathrm{FU}, \mathrm{Au}-\mathrm{Ag} \mathrm{NCs}$ results in ROS (reactive oxygen species) triggered apoptosis mediated cell death. Further, luminescence of $\mathrm{Au}-\mathrm{Ag} \mathrm{NCs}$ serves to track the gene delivery into cells. The detailed mechanism of uptake and manner of cell death has been demonstrated to comprehend the combinatorial therapeutic efficacy of the composite system.

Researchers at JNCASR, Bangalore, demonstrated glucose-derived carbon nanospheres nuclear targeting vector (Selvi et al., 2008). Besides, the intrinsic fluorescence property of carbon nanospheres helps in tracking their cellular localization without any additional fluorescent tags. The spheres are found to target the nucleus of the mammalian cells, causing no toxicity. Interestingly, the in vivo experiments show that these nanospheres have an important ability to cross the blood-brain barrier and localize in the brain besides getting localized in the liver and the spleen. There is also evidence to show that they are continuously being removed from these tissues over time. Furthermore, these nanospheres were used as a carrier for the membraneimpermeable molecule CTPB (N-(4-chloro-3trifluoromethylphenyl)-2-ethoxybenzamide), the only known small-molecule activator of histone acetyltransferase (HAT) p300. Biochemical analyses

\section{References}

Bhatnagar P, Pant A B, Shukla Y, Chaudhari B, Kumar P and Gupta K C (2015) Bromelain nanoparticles protect against 7,12-dimethylbenz[a]anthracene induced skin carcinogenesis in mouse model Eur J Pharm Biopharm 91 $35-46$

Bhowmick S and Koul V (2016) Assessment of PVA/silver such as Western blotting, immunohistochemistry, and gene expression analysis show the induction of hyperacetylation of histone acetyltransferase (HAT) p300 (autoacetylation) as well as histones both in vitro and in vivo and the activation of HAT-dependent transcription upon CTPB delivery. These results establish an alternative path for the activation of gene expression mediated by the induction of HAT activity instead of histone deacetylase (HDAC) inhibition.

\section{Nanoformulations as Antimicrobial Scaffold}

Researchers at IIT, Delhi, presented a novel, elastic, non-adhesive and antimicrobial hydrogel PVA scaffold (loaded with AgNPs) that were synthesized using freeze-thaw method (Bhowmick et al., 2016). The direct visualization of the synthesized (one-pot green synthesis methodology) AgNPs using TEM shows particle size in the range of $7 \pm 3 \mathrm{~nm}$. The minimum inhibitory concentration (MIC) of AgNPs for Staphylococcus aureus and Escherichia coli was estimated to be $7.81 \mu \mathrm{g} / \mathrm{mL}$, whereas for Pseudomonas aeruginosa (gram negative) it was around $3.90 \mu \mathrm{g} / \mathrm{mL}$. The antimicrobial efficacy of AgNPs was further studied by protein leakage, ROS and LDH activity assay. The quantitative elemental analysis of silver was calculated before and after release in phosphate buffer ( $\mathrm{pH}-7.4)$ by atomic absorption spectroscopy. The antimicrobial efficacy of the scaffold was retained even after $96 \mathrm{hr}$ of release of AgNPs which suggests that the scaffold can be used as a reservoir for AgNPs to maintain a moist and sterile environment for a long period of time.

\section{Acknowledgement}

The authors acknowledge Dr. Priyanka Bhatnagar, INSPIRE Faculty, for collecting research contributions from respective researchers and compilation of the information.

nanocomposite hydrogel patch as antimicrobial dressing scaffold: Synthesis, characterization and biological evaluation Mater Sci Eng C Mater Biol Appl 109-119

Coated polymeric nanoparticulate system for multi-drug delivery and development of the same. Indian Patent Application \#1141/DEL/2014

Dalela M, Shrivastav T G, Kharbanda S and Singh H (2015) pH- 
Sensitive biocompatible nanoparticles of paclitaxelconjugated poly(styrene-co-maleic acid) for anticancer drug delivery in solid tumors of syngeneic mice. ACS Appl Mater Interfaces 7 26530-48.

Deepanjalee D, Arun C, Siddhartha SG (2016) Cationic BSA Templated Au-Ag bimetallic nanoclusters as a theranostic gene delivery vector for HeLa cancer cells ACS Biomater Sci Eng 2 2090-2098

Deshpande S, Sharma S, Koul V and Singh N (2017) Core/shell nanoparticles as an efficient sustained-triggered drug delivery system ACS Omega 2 6455-6463

Dhapte V, Kadam S, Moghe A and Pokharkar V (2014) Probing the wound healing potential of biogenic silver nanoparticles $J$ Wound Care 23 431-2

Dinda A K, Bhat M, Kottarath S K and Prashant C K (2016) Novel nanocarrier for oral Hepatitis B vaccine Vaccine $\mathbf{3 4}$ 3076-81

Dinda A K, Bhat M and Sarath K (2016) Novel nanocarrier Amphotericin B-pharmacokintic and pharmoacodynamic analysis. Conference Proceedings; Keystone Symposium: Drug Discovery for Parasitic Disease (A5) 101

Fatima M T, Chanchal A, Yavvari P S, Bhagat S D, Gujrati M, Mishra R K, Srivastava A (2016) Cell permeating nanocomplexes of amphiphilic polyelectrolytes enhance solubility, stability, and anti-cancer efficacy of curcumin Biomacromolecules 201617 2375-83

Gopikrishna J P, Prasanth B P, Nair S K Menon D (2017) Influence of surface passivation of 2-Methoxyestradiol loaded PLGA nanoparticles on cellular interactions, pharmacokinetics and tumour accumulation Cell Sur B: Biointer $150242-$ 249

Guiseppi A E, Rub AA and Nikhil R K S (2010) SAM- modified microdisc electrode array (mdeas) with functionalised carbon nanotubes Electrochimica Acta 55 4247-4255

Guha R, Chowdhury S, Palui H, Mishra A, Basak S, Mandal T K, Hazra S and Konar A (2013) Doxorubicin-loaded MePEG-PCL nanoparticles for prevention of posterior capsular opacification Nanomedicine (Lond) 8 1415-1428

Gupta N, Panwar A, Kumar R, Sharma S K, Sharma R K and Agrawal V (2013) Green synthesis of silver nanoparticles and their antibacterial activity against multi-drug resistant human pathogens Advan Sci Engingg Med 5 355-361

Harshad H, Ashish K A and Sanyog Jain (2014) Development of stabilized glucomannosylated chitosan nanoparticles using tandem crosslinking method for oral vaccine delivery Nanomedicine 9 2511-29

Kumar D, Kumar G and Das R (2017) Strong larvicidal potential of silver nanoparticles (AgNPs) synthesized using Holarrhena antidysenterica bark extract against malarial vector, Anopheles stephensi J Trace Elem Med Biol (Under revision)

Kumar M, Singh G, Sharma S, Gupta D, Bansal V, Arora V, Bhat M, Srivastava S K, Sapra S, Kharbanda S, Dinda A K and Singh H (2014) Intracellular delivery of peptide cargos using iron oxide based nanoparticles: studies on antitumor efficacy of a BCL-2 converting peptide NuBCP-9 Nanoscale 6 14473-83

Malarvizhi G L, Retnakumari A P, Nair S and Koyakutty M (2014) Transferrin targeted core-shell nanomedicine for combinatorial delivery of doxorubicin and sorafenib against hepatocellular carcinoma Nanomedicine 8 649-59

Nirparaj P, Rajdeep G, Sushovan C, Nandi S, Konar A and Hazra $S$ (2015) Curcumin nanoparticles inhibit corneal neovascularisation J Mol Med 93 1095-106

Pahuja R, Seth K, Shukla A, Shukla R K, Bhatnagar P, Chauhan L K, Saxena P N, Arun J, Chaudhari B P, Patel D K, Singh S P, Shukla R, Khanna V K, Kumar P, Chaturvedi R K and Gupta K C (2015) Trans-blood brain barrier delivery of dopamine-loaded nanoparticles reverses functional deficits in Parkinsonian rats ACS Nano 9 4850-71

Prashant C K, Bhat M, Srivastava S K, Saxena A, Kumar M, Singh A, Samim M, Ahmad F J and Dinda A K (2014) Fabrication of nanoadjuvant with poly-å-caprolactone (PCL) for developing a single-shot vaccine providing prolonged immunity Int J Nanomedicine 9 937-50

Ramachandran R, Junnuthula V R, Gowd G S, Ashokan A, Thomas J, Peethambaran R, Thomas A, Unni A K, Panikar D, Nair S V and Koyakutty M (2017) Theranostic 3Dimensional nano brain-implant for prolonged and localized treatment of recurrent glioma Sci Rep 743271

Ravi V, Kumar S T, Prasanthi B and Ratna J V (2015) Formulation and characterization of pyrazinamide polymeric nanoparticles for pulmonary tuberculosis: Efficiency for alveolar macrophage targeting Ind J Pharm Sci 77 258-266

Selvi B R, Jagadeesan D, Suma B S, Nagashankar G, Arif M, Balasubramanyam K, Eswaramoorthy M and Kundu T K (2008) Intrinsically fluorescent carbon nanospheres as a nuclear targeting vector: Delivery of membraneimpermeable molecule to modulate gene expression in vivo Nano Lett $103182-8$

Sharma A, Kundu S, Reddy A, Bajaj A and Srivastava A (2013) Design and engineering of disulfide crosslinked nanocomplexes of polyamide polyelectrolytes: Stability under biorelevant conditions and potent cellular internalization of entrapped model peptide Macromol 
Biosci 13 927-37

Sharma D, Sharma R K, Bhatnagar A, Nishad D K, Singh T, Gabrani R, Sharma S K, Ali J and Dang S (2016) Nose to brain delivery of midazolam loaded PLGA nanoparticle: In vitro and in vivo investigations Cur Drug Del 13 55764

Sharma D, Sharma R K, Sharma N, Gabrani R, Sharma S K, Ali J and Dang S (2015) Nose to brain delivery of PLGAdiazepam nanoparticles AAPS Pharm Sci Tech 16 1108-21

Shukla N K, Padmaker P and Anamika P (2017) Surface modification by self assembled monolayer and carbon nanotubes Emerging Mat Res 6 15-20

Singh S, Upadhyay M, Sharma J, Gupta S, Vivekanandan P and Elangovan R (2016) A portable immunomagnetic cell capture system to accelerate culture diagnosis of bacterial infections Analyst 141 3358-66

Singh S, Upadhyay M, Pandey V, Vivekanandan P, Gupta S and Elangovan R (2016) Spot immunomagnetic enrichment device for rapid detection of pathogens in peripheral blood Adv Mater Technol 1600101

Smitha K T, Sreelakshmi M, Nisha N, Jayakumar R and Biswas R (2014) Amidase encapsulated O-carboxymethyl chitosan nanoparticles for vaccine delivery Int J Biol Macromol 63 $154-7$

Thimmaraju M K, Bheemanapally K, Dharavath R, Kakarla L and Botlagunta M (2017) Improved anticancer activity of meloxicam hydrogels in K562 and HL60 cell lines J Young Pharm 9 209-213
Tiwari M, Narayanan K, Thakar M B, Jagani H V and Venkata Rao J (2014) Biosynthesis and wound healing activity of copper nanoparticles IET Nanobiotechnol 8 230-7

Tiwari S K, Agarwal S, Seth B, Yadav A, Nair S, Bhatnagar P, Karmakar M, Kumari M, Chauhan LK, Patel D K, Srivastava V, Singh D, Gupta S K, Tripathi A, Chaturvedi R K and Gupta K C (2014) Curcumin-loaded nanoparticles potently induce adult neurogenesis and reverse cognitive deficits in Alzheimer's disease model via canonical Wnt/âcatenin pathway ACS Nano 8 76-103

Want M Y, Islamuddin M, Chouhan G, Ozbak H A, Hemeg H A, Dasgupta A K, Chattopadhyay A P and Afrin F (2015) Therapeutic efficacy of artemisinin-loaded nanoparticles in experimental visceral leishmaniasis Cell Sur B Biointer $130215-21$

Patent: Method of developing glutathione stabilized/functionalized collagen nanoparticles Indian Patent Application \#201711007245

Patent: Novel nanocarrier for efficient drug delivery. Indian Patent Application \#201611009768

Patent: Method of developing glutathione stabilized/functionalized collagen nanoparticles. Indian Patent Application \#201711007245

Patent: A process for preparation of non-viral vector for delivery of nucleic acid by mucosal route. Indian Patent Application \# 3256/DEL/2012. 